\title{
Weakly Orthogonal Spherical Harmonics in Non-Polar Spherical Coordinates and its Application to Functions on Cubed-Sphere
}

\author{
M.A.A.M. Faham ${ }^{*}$ and H.M. Nasir ${ }^{* *}$
}

*Department of Mathematics, South Eastern University, Sri Lanka, Email: faham75@yahoo.com

${ }^{* *}$ Department of Mathematics, University of Peradeniya, Sri Lanka, Email: nasirh@pdn.ac.lk

\begin{abstract}
In a recent paper a set of weakly orthogonal and completely orthogonal spherical harmonics in a non-polar spherical coordinate system based on a cubed-sphere was constructed. In this work, we explore some linear relations between these two sets of spherical harmonics. Moreover, a power representation for the set of weakly orthogonal spherical harmonics corresponding to a mode is presented. We also determine the norm of the orthogonal spherical harmonics and hence the inner products for the weakly orthogonal spherical harmonics. As an immediate application of these properties, we present a Fourier series formulation of spherical functions defined on the cubedsphere.
\end{abstract}

KEYWORDS: Non-polar spherical coordinate system, Cubed sphere, Spherical harmonics.

$$
\text { التو افقيات الكروية ضعيفة التعامد في إحداثيات كروية غير قطبية وتطبيقاتها على دوال في مكعب كروي }
$$

محمد فهم و حنيفة ناصر

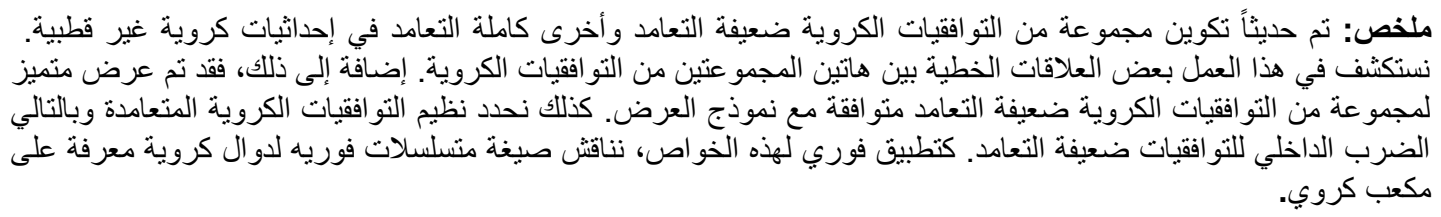

\section{Introduction}

Jumerical computations on the sphere in solving problems defined on the sphere suffer from many 1 difficulties near the poles with a spherical polar coordinate system for the spherical surface. For example, in the computations of global weather prediction models, concentrated grid points near the poles increase the amount of computations in the pole region where quantities of interest are of less importance than in other parts 


\section{WEAKLY ORTHOGONAL SPHERICAL HARMONICS}

of the globe (Nasir, 2007), non-smoothness and unbounded derivatives of bounded functions on the sphere at the poles (Gomez et al., 1997; Phillips, 1957; Reisswig et al., 2007; Rockmore et al., 2003) and unbounded entries in the matrices of finite element approximations (Swartzrauber, 1979,1981) are some example problems faced near the poles. These are collectively called the 'pole problems'.

Searching for remedies for pole problems have attracted some researches in the recent past (Archibald et al., 2011; Chen and Xiao, 2008; Gomez et al., 1997; Nasir, 2007; Nasir and Kako, 2001; Nasir et al., 2003; Rockmore at al., 2003; Ronchi et al., 1996; Swartzrauber, 1979,1981). One of the developments in this direction is to define a grid mesh on the sphere that does not contain polar concentrated points. Among these the 'cubedsphere' defined from the surface of a unit cube has been used by some authors for approximating weather prediction models by finite difference and finite element methods (Phillips, 1957; Reisswig et al., 2007; Ronchi et al., 1996). For some latest papers in this direction, see for example Archibald et al. (2011), Chen and Xiao (2008) and Lauritzen et al. (2010).

In a recent paper Nasir (2007), one of the present authors has defined a non-polar coordinate system based on the cubed-sphere concept and constructed sets of weakly orthogonal and completely orthogonal spherical harmonic functions. These spherical harmonics can be used as basis functions for representing functions defined on the cubed-sphere by Fourier series expansion. In the Fourier series expansion, one encounters the evaluation of inner products of these basis functions. In Nasir (2007), numerical quadrature was used to approximately compute these inner products.

In this paper, we establish more properties and results for the weakly orthogonal and completely orthogonal spherical harmonics. We use these properties to obtain explicit formulas for inner products of the completely orthogonal and hence weakly orthogonal spherical harmonics on the cubed-sphere.

The rest of the paper is organized as follows: Section 2 recalls the results in Nasir (2007) and establishes some notations and conventions for the spherical harmonics. Section 3 presents a power representation for the weakly orthogonal spherical harmonics. Section 4 determines the inner product for the orthogonal spherical harmonics. Section 5 establishes some linear relations between the weakly orthogonal and orthogonal spherical harmonics. Section 6 gives a Fourier series form using the weakly orthogonal spherical harmonics and finally, Section 7 draws a conclusion.
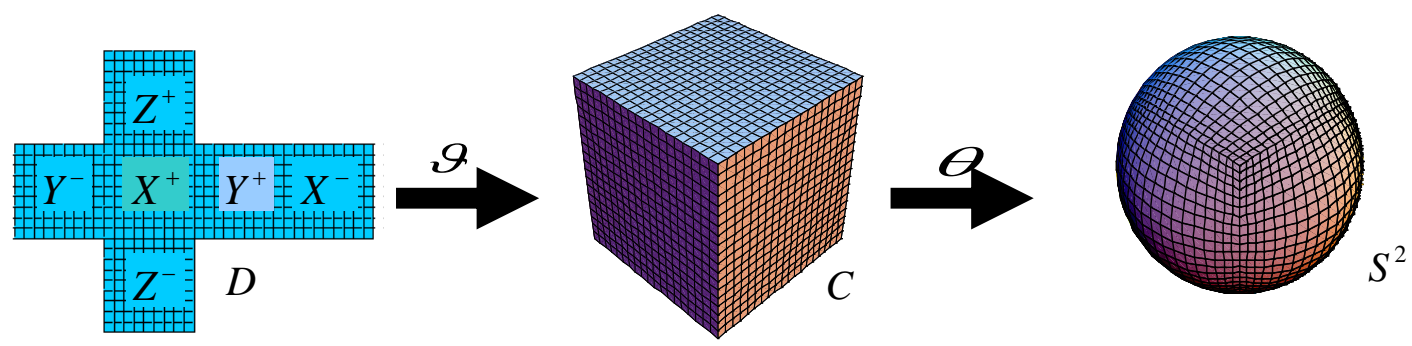

Figure 1. Mapping from the cube surface to spherical surface.

\section{Preliminaries and notations}

In this section, we briefly list the main results presented in Nasir (2007). Let $C$ be the surface of the cube $[-1,1]^{3}$ in the $x y z$ coordinate system. The surface $C$ of the cube with its six faces of the cube are denoted by the six-tuple $C=(X+, Y+, Z+, X-, Y-, Z-)$, where, for example, $X+$ denotes the face across the positive $x$ axis. 


\section{M.A.A.M. FAHAM and H.M. NASIR}

Each face of the cube is assigned a two-dimensional local coordinate system. The cube is then transformed to the unit sphere $S^{2}$ by a mapping (Figure 1). This divides the surface of the unit sphere into six 'faces', each of which corresponds to a face on the cube.

The cubed-spherical coordinate system for the three dimensional space is defined as a six-tuple of the local coordinate systems for the cube faces and is given by $\phi\left(r, t_{1}, t_{2}\right)=r \theta\left(\vartheta\left(t_{1}, t_{2}\right)\right)$, where $\vartheta$ is the mapping from the domain $D$ of two-dimensional faces to the cube and is given by

$$
\vartheta\left(t_{1}, t_{2}\right)=\left\{\left(1, t_{1}, t_{2}\right),\left(-t_{1}, 1, t_{2}\right),\left(-t_{2}, t_{1}, 1\right),\left(-1,-t_{1}, t_{2}\right),\left(t_{1},-1, t_{2}\right),\left(t_{2}, t_{1},-1\right)\right\},-1 \leq t_{1}, t_{2} \leq 1,
$$

$r$ is the standard radial coordinate and $\theta$ is the transformation mapping from the cube to the sphere.

The coordinate signs are chosen according the orientation of the local coordinate systems as shown in Figure 2. Each triplet in the six-tuple is defined on the six faces of the cubed-sphere by $\theta(\mathbf{p})=\mathbf{p} /\|\mathbf{p}\|, \mathbf{p} \in C$. The function $\theta$ maps each face on $C$ to a corresponding surface on the sphere $S^{2}$. For convenience, where there is no ambiguity, these mapped faces on the sphere are also denoted by $S^{2}=(X+, Y+, Z+, X-, Y-, Z-)$. For a detailed discussion on the construction of the cubed-spherical coordinate system, we refer to Nasir (2007).
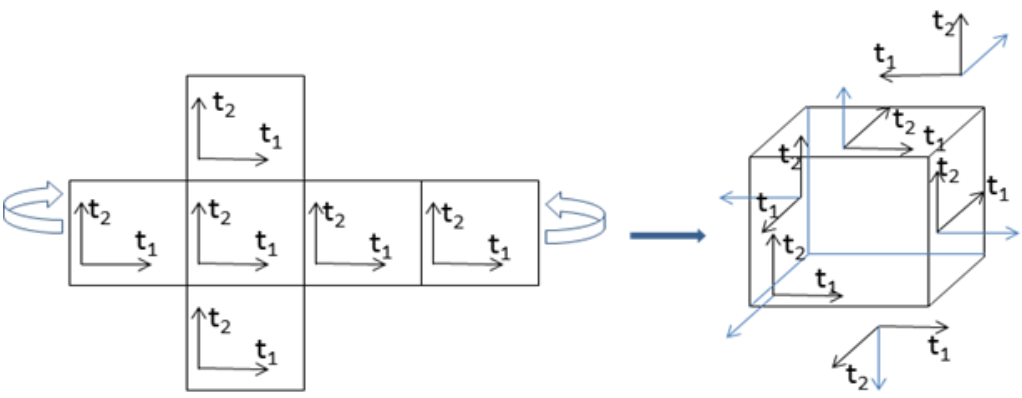

Figure 2. Orientation of local coordinate parameters.

A spherical function defined on the unit 2-sphere $S^{2}$ is described in the cubed-spherical coordinates as a six-tuple of functions defined for each face with respect to its local coordinates

$$
f\left(t_{1}, t_{2}\right):=\left(f_{X_{+}}\left(t_{1}, t_{2}\right), f_{Y+}\left(t_{1}, t_{2}\right), f_{Z_{+}}\left(t_{1}, t_{2}\right), f_{X_{-}}\left(t_{1}, t_{2}\right), f_{Y_{-}}\left(t_{1}, t_{2}\right), f_{Z_{-}}\left(t_{1}, t_{2}\right)\right) .
$$

The Laplace-Beltrami operator on the unit 2-sphere is given by

$$
\Delta_{S}=s_{t}\left(\left(1+t_{1}^{2}\right) \frac{\partial^{2}}{\partial t_{1}^{2}}+2 t_{1} t_{2} \frac{\partial^{2}}{\partial t_{1} \partial t_{2}}+\left(1+t_{2}^{2}\right) \frac{\partial^{2}}{\partial t_{2}^{2}}+2 t_{1} \frac{\partial}{\partial t_{1}}+2 t_{2} \frac{\partial}{\partial t_{2}}\right)
$$

for all faces of $S^{2}$, where $s_{t}=1+t_{1}^{2}+t_{2}^{2}$. The eigenvalue problem for spherical harmonics is given by

$$
\Delta_{S} u=-l(l+1) u
$$

where $l$ is an integer.

\subsection{Spherical harmonics}

A set of linearly independent solutions for the eigenvalue problem (2) is given by

$$
y_{l}^{(m, n)}=\frac{p_{l}^{(m, n)}}{s_{t}^{l / 2}}, \quad m+n=l-1, l
$$

where $p_{l}^{(m, n)}\left(t_{1}, t_{2}\right)$ are polynomials satisfying the differential equation 


\section{WEAKLY ORTHOGONAL SPHERICAL HARMONICS}

$$
\left(1+t_{1}^{2}\right) \frac{\partial^{2} P}{\partial t_{1}^{2}}+2 t_{1} t_{2} \frac{\partial^{2} P}{\partial t_{1} \partial t_{2}}+\left(1+t_{2}^{2}\right) \frac{\partial^{2} P}{\partial t_{2}^{2}}-(l-1)\left(2 t_{1} \frac{\partial P}{\partial t_{1}}+2 t_{2} \frac{\partial P}{\partial t_{2}}-l P\right)=0 .
$$

The polynomials $p_{l}^{(m, n)}$ are explicitly given by the non-zero real or imaginary parts of

$$
p_{l}^{(m, n)}\left(t_{1}, t_{2}\right)=\sum_{p=m_{1}}^{m} 2 \sum_{q=n_{1}}^{n} 2\left(\begin{array}{c}
(m-p+n-q) / 2 \\
(m-p) / 2
\end{array}\right)\left(\begin{array}{c}
l \\
p, q
\end{array}\right) t_{1}^{p} t_{2}^{q} \mathrm{i}^{(l-p-q)}, \quad m+n=l-1, l,
$$

where $m_{1}=m \bmod 2, n_{1}=n \bmod 2, \mathrm{i}=\sqrt{-1}$, the subscript 2 in the summation notation indicates that the index variables increase with step 2, and $\left(\begin{array}{l}a \\ b\end{array}\right)=\frac{a !}{b !(a-b) !}$ and $\left(\begin{array}{c}a \\ b, c\end{array}\right)=\frac{a !}{b ! c !(a-b-c) !}$ are the binomial and trinomial coefficients respectively.

The degree of the polynomial $p_{l}^{(m, n)}\left(t_{1}, t_{2}\right)$ is $(m, n)$ with total degree $m+n$. The leading coefficient of $p_{l}^{(m, n)}$ is $a_{l}^{(m, n)}=\left(\begin{array}{c}l \\ m, n\end{array}\right)$.

The polynomials display many symmetric properties. One property is that they are either odd or even functions with respect to the parameters. That is, $p_{l}^{(m, n)}\left( \pm t_{1}, \pm t_{2}\right)=( \pm 1)^{m}( \pm 1)^{n} p_{l}^{(m, n)}\left(t_{1}, t_{2}\right)$. The same symmetric property holds for the spherical harmonics as well. That is, $y_{l}^{(m, n)}\left( \pm t_{1}, \pm t_{2}\right)=( \pm 1)^{m}( \pm 1)^{n} y_{l}^{(m, n)}\left(t_{1}, t_{2}\right)$. Another symmetric property is that the polynomial gives another independent solution for the same mode when the parameters are switched, $p_{l}^{(n, m)}\left(t_{1}, t_{2}\right)=p_{l}^{(m, n)}\left(t_{2}, t_{1}\right)$. The same holds for the spherical harmonics as well. That is, $y_{l}^{(n, m)}\left(t_{1}, t_{2}\right)=y_{l}^{(m, n)}\left(t_{2}, t_{1}\right)$.

A set of continuous spherical harmonics can be constructed from the eigenfunctions of one face forming a six-tuple of functions

$$
Y\left(t_{1}, t_{2}\right):=\left(y\left(t_{1}, t_{2}\right), y\left(-\frac{1}{t_{1}},-\frac{t_{2}}{t_{1}}\right), y\left(-\frac{t_{1}}{t_{2}},-\frac{1}{t_{2}}\right), y\left(t_{1},-t_{2}\right), y\left(-\frac{1}{t_{1}}, \frac{t_{2}}{t_{1}}\right), y\left(\frac{t_{1}}{t_{2}},-\frac{1}{t_{2}}\right)\right),
$$

where each component in the six-tuple is an eigenfunction obtained from the eigenfunction $y\left(t_{1}, t_{2}\right)$ of the face $X+$. Details of the continuity of $Y\left(t_{1}, t_{2}\right)$ can be found in Nasir (2007). For spherical harmonics in a spherical polar coordination system, see (MacRobert, 1967).

The inner product on the space of square integrable functions $L^{2}\left(S^{2}\right)$ is given by

$$
\langle u, v\rangle_{S}=\sum_{F \in\{X \pm, Y \pm, Z \pm\}}\langle u, v\rangle_{F},
$$

where $\langle u, v\rangle_{F}=\int_{-1}^{1} \int_{-1}^{1} u_{F} v_{F} s_{t}^{-3 / 2} d t_{1} d t_{2}$ are the inner products for the functional components for one face $F \in\{X+, X-, Y+, Y-, Z+, Z-\}$.

The spherical harmonics $Y_{l}^{(m, n)}$ are weakly orthogonal in the sense that they are orthogonal for distinct modes - because the Laplace-Beltrami operator is self-adjoint - but are not orthogonal among the $2 l+1$ functions for a given mode $l$.

\subsection{Completely orthogonal spherical harmonics}

A set of completely orthogonal spherical harmonics can be constructed from the eigensolutions in the form 


\section{M.A.A.M. FAHAM and H.M. NASIR}

$$
Z_{l, r}^{(k)}\left(t_{1}, t_{2}\right):=\frac{Q_{l, r}^{(k)}}{s_{t}^{l / 2}}, \quad r=0,1, \cdots, l-k, \quad k=0,1
$$

with polynomials $Q_{l, r}^{(k)}=p_{r}^{(k)}\left(t_{1}\right) \rho\left(t_{1}\right)^{l-r} q_{l-r}(l, \lambda)$, where $p_{r}^{(k)}\left(t_{1}\right)$ and $q_{l-r}(l, \lambda)$ are polynomials in the variables $t_{1}$ and $\lambda$ respectively with degrees $l-k,(k=0,1)$ and $l-r$ respectively satisfying the differential equations

$$
\left(1+t_{1}^{2}\right) \frac{d^{2} p}{d t_{1}^{2}}-2(r-1) t_{1} \frac{d p}{d t_{1}}+r(r-1) p=0
$$

and

$$
\left(1+\lambda^{2}\right) \frac{d^{2} q}{d \lambda^{2}}-(2 l-1) \lambda \frac{d q}{d \lambda}+(l-r)(l+r) q=0
$$

respectively, and $\rho\left(t_{1}\right)=\left(1+t_{1}^{2}\right)^{1 / 2}, \lambda=t_{2} / \rho\left(t_{1}\right)$.

For orthogonal polynomial solutions of differential equations, see (Krall and Sheffer,1967; Kwon et al., 2001; Lee and Littlejohn, 2006; and the references therein). The differential equations we have in (9) and (10) are slightly different from the equations treated by the authors in those papers. Nevertheless, their approach can be directly applicable to equations (9) and (10).

The polynomials $p_{r}^{(k)}\left(t_{1}\right)$ can be obtained from $p_{r}^{(0)}+\mathrm{i} p_{r}^{(1)}=\left(t_{1}+\mathrm{i}\right)^{r}$ and the polynomials $q_{l-r}(l, \lambda)$ are given by $q_{l-r}(l, \lambda)=\sum_{n=\eta_{1}}^{l-r} a_{l, n}^{(l-r)} \lambda^{n}$, where the coefficients $a_{l, n}^{(l-r)}$ satisfy the recurrence relation

$$
a_{l, n}^{(l-r)}=-(n+1)(n+2) /(l-r-n)(l+r-n) a_{l, n+2}^{(l-r)}, \quad n=l-r-2, l-r-4, \cdots, r_{1},
$$

where $r_{1}=(l-r) \bmod 2$. The polynomials $q_{l-r}(l, \lambda)$ are normalized such that the leading coefficient $a_{l, r}^{(l-r)}=\left(\begin{array}{c}l \\ l-r\end{array}\right)$. Hence, we get

$$
a_{l, l-n}^{(l-r)}=\frac{(-1)^{(l-r-n) / 2}}{2^{l-r-n}}\left(\begin{array}{c}
l \\
(l-r-n) / 2, n
\end{array}\right), \quad n=l-r-2, l-r-4, \cdots, r_{1} .
$$

The polynomials $Q_{l, r}^{(k)}\left(t_{1}, t_{2}\right)$ have, in expanded form, degrees $(r-k, l-r)$ for with total degree $l-k$.

The orthogonal spherical harmonics are then given by the six-tuple formed by $Z_{l, r}^{(k)}\left(t_{1}, t_{2}\right)$ as in (6). The set of polynomials corresponding to the weakly orthogonal and completely orthogonal spherical harmonics for the face $X+$ are given in Table 1 for the first five modes.

\section{A power representation}

In this section, we establish a representation of the weakly orthogonal spherical harmonics for a given mode $l$ as a monomial power of a simple linear expression.

Theorem 3.1 Let $s, j$ be symbolic (indeterminate) variables connected by the $s-j$ relation $j^{2}=-\left(1+s^{2}\right)$. For a mode $l$, the $2 l+1$ polynomials $p_{l}^{(m, n)}$ in (5) can be given by the coefficients of the powers of $s$ of the expansion of $\left(1+t_{1} s+t_{2} j\right)^{l}=P_{l}^{(1)}(s)+j P_{l}^{(2)}(s)$, where, in the expansion of the trinomial, the even powers of $j$ 


\section{WEAKLY ORTHOGONAL SPHERICAL HARMONICS}

are substituted by the $s-j$ relation.

Proof. Expanding the trinomial power and substituting the $s$-j relations for even powers of $j$ we get,

$$
\begin{aligned}
\left(t_{1}+t_{2} s+j\right)^{l} & =\sum_{p+q+r=l}\left(\begin{array}{c}
l \\
p, q
\end{array}\right) t_{1}^{p} t_{2}^{q} s^{q} j^{r} \\
& =\sum_{\substack{p+q+r=l \\
r-\text { even }}}\left(\begin{array}{c}
l \\
p, q
\end{array}\right) t_{1}^{p} t_{2}^{q} s^{q} j^{r}+j \sum_{\substack{p+q+r=l \\
r-o d d}}\left(\begin{array}{c}
l \\
p, q
\end{array}\right) t_{1}^{p} t_{2}^{q} s^{q} j^{r-1}=: P_{l}^{(1)}+j P_{l}^{(2)} .
\end{aligned}
$$

Considering the first term with even $r$,

$$
P_{l}^{(1)}(s)=\sum_{\substack{p+q+r=l \\
r-\text { even }}}\left(\begin{array}{c}
l \\
p, q
\end{array}\right) t_{1}^{p} t_{2}^{q} s^{q} \mathbf{i}^{r}\left(1+s^{2}\right)^{r / 2}=\sum_{\substack{p+q+r=l \\
r-\text { even }}} \sum_{k=0}^{r / 2}\left(\begin{array}{c}
l \\
p, q
\end{array}\right)\left(\begin{array}{c}
r / 2 \\
k
\end{array}\right) t_{1}^{p} t_{2}^{q} \mathbf{i}^{r} s^{q+2 k} .
$$

Now, collecting the coefficients of $s^{n}$ where $n=q+2 k$ or $k=(n-q) / 2$, and noting that $n$ and $q$ must be of same parity for $k$ to be an integer and hence that $m$ and $p$ must be of same parity, we have

$$
P_{l}^{(1)}(s)=\sum_{n=0}^{l}\left(\sum_{p=m_{1}}^{m} 2 \sum_{q=n_{1}}^{n} 2\left(\begin{array}{c}
l \\
p, q
\end{array}\right)\left(\begin{array}{c}
(m+n-p-q) / 2 \\
(n-q) / 2
\end{array}\right) t_{1}^{p} t_{2}^{q} \mathbf{i}^{l-p-q}\right) s^{n}, m+n=l,
$$

where $m_{1}=m \bmod 2$ and $n_{1}=n \bmod 2$ to force the parity of $p$ and $q$ to conform with that of those $m$ and $n$ respectively.

Similarly, for the second term, we have

$$
P_{l}^{(2)}(s)=\sum_{n=0}^{l-1}\left(\sum_{p=m_{1}}^{m} 2 \sum_{q=n_{1}}^{n} 2\left(\begin{array}{c}
l \\
p, q
\end{array}\right)\left(\begin{array}{c}
(m+n-p-q) / 2 \\
(n-q) / 2
\end{array}\right) t_{1}^{p} t_{2}^{q} \mathbf{i}^{l-p-q}\right) s^{n}, m+n=l-1 .
$$

The coefficients of $P_{l}^{(1)}(s)$ and $P_{l}^{(2)}(s)$ are the polynomials $p_{l}^{(m, n)}$ for $m+n=l-1, l$.

We now show that the polynomials can be computed by the convolution products of lower order polynomials.

Theorem 3.2 Let $\left(t_{1}+t_{2} s+j\right)^{l}=P_{l}^{(1)}(s)+j P_{l}^{(2)}(s)$. Then the polynomials for mode $l_{1}+l_{2}$ are given by

$$
P_{l_{1}+l_{2}}^{(1)}=P_{l_{1}}^{(2)} P_{l_{2}}^{(2)}-\left(1+s^{2}\right) P_{l_{1}}^{(2)} P_{l_{2}}^{(2)} \text { and } P_{l_{1}+l_{2}}^{(2)}=P_{l_{1}}^{(1)} P_{l_{2}}^{(2)}+P_{l_{1}}^{(2)} P_{l_{2}}^{(1)} \text {. }
$$

Proof. Expansion of the powers gives

$\left(t_{1}+t_{2} s+j\right)^{l_{1}+l_{2}}=\left(P_{l_{1}}^{(1)}+j P_{l_{1}}^{(2)}\right)\left(P_{l_{2}}^{(1)}+j P_{l_{2}}^{(2)}\right)=P_{l_{1}}^{(1)} P_{l_{2}}^{(1)}-\left(1+s^{2}\right) P_{l_{1}}^{(2)} P_{l_{2}}^{(2)}+j\left(P_{l_{1}}^{(1)} P_{l_{2}}^{(2)}+P_{l_{1}}^{(2)} P_{l_{2}}^{(1)}\right)$.

Again we have $\left(t_{1}+t_{2} s+j\right)^{l_{1}+l_{2}}=P_{l_{1}+l_{2}}^{(1)}+j P_{l_{1}+l_{2}}^{(2)}$. Equate the coefficients of $j$.

The polynomial multiplications are the convolution products involving the coefficients $p_{l}^{(m, n)}, m+n=l$ for $P_{l}^{(1)}$ and $p_{l}^{(m, n)}, m+n=l-1$ for $P_{l}^{(2)}$.

Thus, if we denote the sequence of coefficients of the polynomials with the same notation, we have

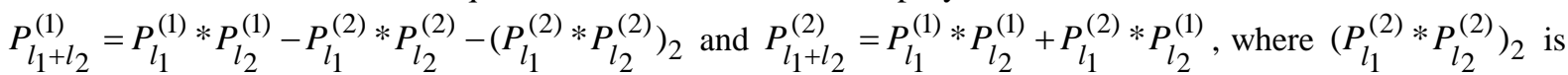
the sequence shifted two places to the right. 


\section{M.A.A.M. FAHAM and H.M. NASIR}

Table 1. Polynomials for weakly orthogonal and completely orthogonal spherical harmonics (SH).

\begin{tabular}{|c|c|c|c|c|}
\hline$l$ & $(m, n)$ & $\begin{array}{l}\text { Polynomials } P_{l}^{(m, n)}\left(t_{1}, t_{2}\right) \\
\text { for weakly orthogonal } \mathrm{SH}\end{array}$ & {$[r, k]$} & $\begin{array}{l}\text { Polynomials } Q_{l, r}^{(k)}\left(t_{1}, t_{2}\right) \text { for completely } \\
\text { orthogonal SH }\end{array}$ \\
\hline 0 & $(0,0)$ & 1 & {$[0,0]$} & 1 \\
\hline 1 & $\begin{array}{l}(1,0) \\
(0,0) \\
(0,1)\end{array}$ & $\begin{array}{l}t_{1} \\
1 \\
t_{2}\end{array}$ & $\begin{array}{l}{[1,1]} \\
{[0,1]} \\
{[1,0]}\end{array}$ & $\begin{array}{l}t_{1} \\
1 \\
t_{2}\end{array}$ \\
\hline 2 & $\begin{array}{l}(1,0) \\
(0,1) \\
(2,0) \\
(1,1) \\
(0,2)\end{array}$ & $\begin{array}{l}2 t_{1} \\
2 t_{2} \\
-1+t_{1}^{2} \\
2 t_{1} t_{2} \\
-1+t_{2}^{2}\end{array}$ & $\begin{array}{l}{[2,1]} \\
{[1,1]} \\
{[2,0]} \\
{[1,0]} \\
{[0,0]}\end{array}$ & $\begin{array}{l}2 t_{1} \\
2 t_{2} \\
-1+t_{1}^{2} \\
2 t_{1} t_{2} \\
-1 / 2-t_{1}^{2} / 2+t_{2}^{2}\end{array}$ \\
\hline 3 & $\begin{array}{l}(2,0) \\
(1,1) \\
(0,2) \\
(3,0) \\
(2,1) \\
(1,2) \\
(0,3)\end{array}$ & $\begin{array}{l}-1+3 t_{1}^{2} \\
6 t_{1} t_{2} \\
-1+3 t_{2}^{2} \\
-3 t_{1}+t_{1}^{3} \\
-3 t_{2}+3 t_{1}^{2} t_{2} \\
-3 t_{1}+3 t_{1} t_{2}^{2} \\
-3 t_{2}+t_{2}^{3}\end{array}$ & $\begin{array}{l}{[3,1]} \\
{[2,1]} \\
{[1,1]} \\
{[3,0]} \\
{[2,0]} \\
{[1,0]} \\
{[0,0]}\end{array}$ & $\begin{array}{l}-1+3 t_{1}^{2} \\
6 t_{1} t_{2} \\
-3 / 4-3 t_{1}^{2} / 4+3 t_{2}^{2} \\
-3 t_{1}+t_{1}^{3} \\
-3 t_{2}+3 t_{1}^{2} t_{2} \\
-3 t_{1} / 4-3 t_{1} t_{2}^{2} / 4+3 t_{1} t_{2}^{2} \\
-3 t_{2} / 2+3 t_{1}^{2} t_{2} / 2+t_{2}^{3}\end{array}$ \\
\hline 4 & $\begin{array}{l}(3,0) \\
(2,1) \\
(1,2) \\
(0,3) \\
(4,0) \\
(3,1) \\
(2,2) \\
(1,3) \\
(0,4)\end{array}$ & $\begin{array}{l}-4 t_{2}+4 t_{1}^{3} \\
-4 t_{2}+12 t_{1}^{2} t_{2} \\
-4 t_{1}+12 t_{1} t_{2}^{2} \\
-4 t_{2}+4 t_{2}^{3} \\
1-6 t_{1}^{2}+t_{1}^{4} \\
-12 t_{1} t_{2}+4 t_{1}^{3} t_{2} \\
2-6 t_{1}^{2}-6 t_{2}^{2}+6 t_{1}^{2} t_{2}^{2} \\
-12 t_{1} t_{2}+4 t_{1} t_{2}^{3} \\
1-6 t_{2}^{2}+t_{2}^{4}\end{array}$ & $\begin{array}{l}{[4,1]} \\
{[3,1]} \\
{[2,1]} \\
{[1,1]} \\
{[4,0]} \\
{[3,0]} \\
{[2,0]} \\
{[1,0]} \\
{[0,0]}\end{array}$ & $\begin{array}{l}-4 t_{2}+4 t_{1}^{3} \\
-4 t_{2}+12 t_{1}^{2} t_{2} \\
-2 t_{1}-2 t_{1}^{3}-12 t_{1} t_{2}^{2} \\
-3 t_{2}-3 t_{1}^{2} t_{2}+4 t_{2}^{3} \\
1-6 t_{1}^{2}+t_{1}^{4} \\
-12 t_{1} t_{2}+4 t_{1}^{2} t_{2} \\
1-t_{1}^{4}-6 t_{2}^{2}+6 t_{1}^{2} t_{2}^{2} \\
-3 t_{1} t_{2}-3 t_{1}^{3} t_{2}+4 t_{1} t_{2}^{3} \\
3 / 8+3 t_{1}^{2} / 4+3 t_{1}^{4} / 8-3 t_{2}^{2}-3 t_{1}^{2} t_{2}^{2}+4 t_{2}^{4}\end{array}$ \\
\hline 5 & $\begin{array}{l}(4,0) \\
(3,1) \\
(2,2) \\
(1,3) \\
(0,4) \\
(5,0) \\
(4,1) \\
(3,2) \\
(2,3) \\
(1,4) \\
(0,5)\end{array}$ & $\begin{array}{l}1-10 t_{1}^{2}+5 t_{1}^{4} \\
-20 t_{1} t_{2}+20 t_{1}^{3} t_{2} \\
2-10 t_{1}^{2}-10 t_{2}^{2}+30 t_{1}^{2} t_{2}^{2} \\
-20 t_{1} t_{2}+20 t_{1} t_{2}^{3} \\
1-10 t_{2}^{2}+5 t_{2}^{4} \\
5 t_{1}-10 t_{1}^{3}+t_{1}^{5} \\
5 t_{2}-30 t_{1}^{2} t_{2}+5 t_{1}^{4} t_{2} \\
10 t_{1}-10 t_{1}^{3}-30 t_{1} t_{2}^{2}+10 t_{1}^{3} t_{2}^{2} \\
10 t_{2}-30 t_{1}^{2} t_{2}-10 t_{2}^{3}+10 t_{1}^{2} t_{2}^{3} \\
5 t_{1}-30 t_{1} t_{2}^{2}+5 t_{1} t_{2}^{4} \\
5 t_{2}-10 t_{2}^{3}+t_{2}^{5}\end{array}$ & $\begin{array}{l}{[5,1]} \\
{[4,1]} \\
{[3,1]} \\
{[2,1]} \\
{[1,1]} \\
{[5,0]} \\
{[4,0]} \\
{[3,0]} \\
{[2,0]} \\
{[1,0]} \\
{[0,0]}\end{array}$ & $\begin{array}{l}1-10 t_{1}^{2}+5 t_{1}^{4} \\
-20 t_{1} t_{2}+20 t_{1}^{3} t_{2} \\
5 / 4-5 t_{1}^{2} / 2-5 t_{1}^{4} / 4-10 t_{2}^{2}+30 t_{1}^{2} t_{2}^{2} \\
-10 t_{1} t_{2}-10 t_{1}^{3} t_{2}+20 t_{1} t_{2}^{3} \\
5 / 8+5 t_{1}^{2} / 4+5 t_{1}^{4} / 8-15 t_{2}^{2} / 2-15 t_{1}^{2} t_{2}^{2} / 2+5 t_{2}^{4} \\
5 t_{1}-10 t_{1}^{3}+t_{1}^{5} \\
5 t_{2}-30 t_{1}^{2} t_{2}+5 t_{1}^{4} t_{2} \\
15 t_{1} / 4+5 t_{1}^{3} / 2-5 t_{1}^{5} / 4-30 t_{1} t_{2}^{2}+10 t_{1}^{3} t_{2}^{2} \\
5 t_{2}-5 t_{1}^{4} t_{2}-10 t_{2}^{3}+10 t_{1}^{2} t_{2}^{3} \\
5 t_{1} / 8+5 t_{1}^{3} / 4+5 t_{1}^{5} / 8-15 t_{1} t_{2}^{2} / 2-15 t_{1}^{3} t_{2}^{2} / 2+5 t_{1} t_{2}^{4} \\
15 t_{2} / 8-15 t_{1}^{2} t_{2} / 4+15 t_{1}^{4} t_{2} / 8-5 t_{2}^{3}-5 t_{1}^{2} t_{2}^{3}+t_{2}^{5} \\
\end{array}$ \\
\hline
\end{tabular}




\section{WEAKLY ORTHOGONAL SPHERICAL HARMONICS}

\section{Inner products for completely orthogonal spherical harmonics}

In the Fourier series expansion of spherical functions on a cubed-sphere by weakly orthogonal spherical harmonics, one needs to evaluate the inner products of those spherical harmonics. A method for doing this, however, is not explicitly available. The problem is handled in connection with the inner products of the completely orthogonal spherical harmonics.

In this section, we obtain an explicit form for the inner products of the completely orthogonal spherical harmonics. These are then used in the evaluation of the inner products of the weakly orthogonal spherical harmonics by means of the linear relations to be established in Section 5.

The inner product of the completely orthogonal spherical harmonics $Z_{l, r}^{(k)}$ are given by

$$
\left\langle Z_{l, r}^{(k)}, Z_{l, r^{\prime}}^{(k)}\right\rangle_{S}=8\left(\int_{0}^{\infty} \frac{p_{r}^{(k)}\left(T_{1}\right) p_{r^{\prime}}^{(k)}\left(T_{1}\right)}{\rho\left(T_{1}\right)^{r+r^{\prime}+2}} d T_{1}\right)\left(\int_{0}^{\infty} \frac{q_{l-r}(l, \lambda) q_{l-r^{\prime}}(l, \lambda)}{\rho^{2 l+3}(\lambda)} d \lambda\right)
$$

where $r+r^{\prime}$ is even and $k=0,1$. The inner products of the other distinct combinations of the indices $r, r^{\prime}$ vanish (Nasir, 2007).

By the transformation $T_{1}=\tan \theta$, the first integral is evaluated to

$$
\int_{0}^{\infty} \frac{p_{r}^{(k)}\left(T_{1}\right) p_{r^{\prime}}^{(k)}\left(T_{1}\right)}{\rho\left(T_{1}\right)^{r+r^{\prime}+2}} d T_{1}= \begin{cases}0 & r \neq r^{\prime} \\ \pi / 2 & r=r^{\prime}=0 \\ \pi / 4 & r=r^{\prime}>0\end{cases}
$$

which makes $\left\{Z_{l, r}^{(k)}, l=0,1,2, \cdots ; r=0,1,2, \cdots, l-k ; k=0,1\right\}$ a fully orthogonal set of spherical functions.

To evaluate the inner product (12), we now need to evaluate the second integral for $r=r^{\prime}$.

Lemma 4.1 The polynomials $q_{l-r}(l, \lambda), r=0,1,2, \cdots, l$ are orthogonal in the weighted Hilbert space $L^{2}\left(0, \infty, \rho_{0}\right)$, where $\rho_{0}=\left(1+\lambda^{2}\right)^{-(l+1 / 2)}$. Thus, $q_{l-r}(l, \lambda)$ is orthogonal to any lower order polynomials.

Proof. Differentiating (10) $k$ times, we get

$$
\left(1+\lambda^{2}\right) \frac{d^{2} q^{(k)}}{d \lambda^{2}}-(2(l-k)-1) \lambda \frac{d q^{(k)}}{d \lambda}+(l-r-k)(l+r-k) q^{(k)}=0 .
$$

Writing in self-adjoint form, we get $\frac{d}{d \lambda}\left(\rho_{k+1} q^{(k+1)}\right)+(l-r-k)(l+r-k) \rho_{k} q^{(k)}=0$ or

$$
\rho_{k} q^{(k)}=-\frac{1}{(l-r-k)(l+r-k)} \frac{d}{d \lambda}\left(\rho_{k+1} q^{(k+1)}\right), k=0,1,2, \cdots,
$$

where $\rho_{k+1}=\left(1+\lambda^{2}\right) \rho_{k}, k=0,1,2, \cdots, l$ with $\rho_{0}=\left(1+\lambda^{2}\right)^{-(l+1 / 2)}, q=q_{l-r}(l, \lambda)$.

By repeated application of this relation starting with the case $k=0$, we have

$$
\rho_{0} q_{l-r}^{(0)}=\frac{(-1)^{l-r}(2 r) !}{(l-r) !(l+r) !} \frac{d^{l-r}}{d \lambda^{l-r}}\left(\rho_{l-r} q_{l-r}^{(l-r)}\right) .
$$

Since $q_{l-r}=a_{l, l-r}^{(l-r)} \lambda^{l-r}+$ lots, where, and in other places, 'lots' stands for 'lower order terms', we have $q_{l-r}^{(l-r)}=a_{l, l-r}^{(l-r)}(l-r)$ !, which gives the Rodrigue's type formula for $q_{l-r}(l, \lambda)$ : 


\section{M.A.A.M. FAHAM and H.M. NASIR}

$$
q_{l-r}=q_{l-r}(l, \lambda)=\frac{(-1)^{l-r}(2 r) !}{(l+r) !} a_{l, l-r}\left(1+\lambda^{2}\right)^{l+1 / 2} \frac{d^{l-r}}{d \lambda^{l-r}}\left(1+\lambda^{2}\right)^{-(r+1 / 2)} .
$$

For $r \neq r^{\prime}$, multiplying (13) by $q_{l-r^{\prime}}\left(1+\lambda^{2}\right)^{-(l+1 / 2)}$ and integrating over $(0, \infty)$ with repeated integration by parts, we get $\int_{0}^{\infty} q_{l-r} q_{l-r^{\prime}}\left(1+\lambda^{2}\right)^{-(l+1 / 2)} d \lambda=0$. This gives the orthogonality of the polynomials $q_{l-r}(l, \lambda)$.

Since any polynomial of degree $n$ can be expressed as a linear combination of the polynomials $q_{k}(l, \lambda), k=0,1, \cdots, m$, it is orthogonal to any $q_{n}(l, \lambda), n>m$.

We now evaluate the norm of $q_{l-r}(l, \lambda)$. For convenience, let $I_{\alpha}(c)=\int_{0}^{c}\left(1+\lambda^{2}\right)^{-\alpha} d \lambda$ and $J_{\alpha}(c)=\int_{0}^{c}\left(1+\lambda^{2}\right)^{-\alpha} q_{l-r}^{2} d \lambda$. We need to evaluate $J_{r+3 / 2}(\infty)=\int_{0}^{\infty}\left(1+\lambda^{2}\right)^{-3 / 2} q_{l-r}^{2} d \lambda$.

When $r=r^{\prime}$, using the orthogonality of $q_{l-r}$ with lower order polynomials, Rodrigue's type formula (13) and integration by parts, we have

$$
\begin{aligned}
J_{l+1 / 2}(\infty) & =\int_{0}^{\infty}\left(1+\lambda^{2}\right)^{-(l+1 / 2)} q_{l-r}^{2} d \lambda=a_{l, l-r}^{(l-r)} \int_{0}^{\infty} \lambda^{l-r}\left(1+\lambda^{2}\right)^{-(l+1 / 2)} q_{l-r} d \lambda \\
& =\frac{(-1)^{l-r}(2 r) !}{(l+r) !}\left(a_{l, l-r}^{(l-r)}\right)^{2} \int_{0}^{\infty} \lambda^{l-r} \frac{d^{l-r}}{d \lambda^{l-r}}\left(1+\lambda^{2}\right)^{-(r+1 / 2)} d \lambda=\left(a_{l, l-r}^{(l-r)}\right)^{2} \frac{(2 r) !}{(l+r) !}(l-r) ! \int_{0}^{\infty}\left(1+\lambda^{2}\right)^{-(r+1 / 2)} d \lambda \\
& =\left(a_{l, l-r}^{(l-r)}\right)^{2} \frac{(2 r) !}{(l+r) !}(l-r) ! I_{r+1 / 2}(\infty) .
\end{aligned}
$$

For the evaluation of $I_{r+1 / 2}(\infty)$, we use the identity

$$
\frac{d}{d \lambda} \frac{\lambda}{\left(1+\lambda^{2}\right)^{\alpha}}=(1-2 \alpha) \frac{1}{\left(1+\lambda^{2}\right)^{\alpha}}+2 \alpha \frac{1}{\left(1+\lambda^{2}\right)^{\alpha+1}} .
$$

We shall evaluate $J_{r+3 / 2}(\infty)$ separately for $r=0$ and $r \geq 1$. We first consider the case $r \geq 1$.

Integrating (15) over the positive real line $(0, \infty)$, we get the recurrence relation $I_{\alpha+1}(\infty)=(2 \alpha-1) I_{\alpha}(\infty) /(2 \alpha)$ for $\alpha>1 / 2$ and $I_{\alpha+1}(\infty)=I_{3 / 2}(\infty)=1$ when $\alpha=1 / 2$. Hence, by repeated application of this recurrence relation starting with $\alpha=r-1 / 2, r \geq 1$ and ending with $\alpha=1 / 2$, we get $I_{r+1 / 2}(\infty)=2^{2(r-1)}((r-1) !)^{2} I_{3 / 2}(\infty) /(2 r-1) !=2^{2 r-2}((r-1) !)^{2} /(2 r-1) !$. Therefore, (14) becomes, with the substitution of $a_{l, l-r}^{(l-r)}=\left(\begin{array}{c}l \\ l-r\end{array}\right)$,

$$
J_{l+1 / 2}(\infty)=\frac{\left(a_{l^{\prime} l-r}^{(l-r)}\right)^{2} 2^{2 r-1}}{r\left(\begin{array}{c}
l \\
l-r
\end{array}\right)^{2}} \frac{(l !)^{2}}{(l-r) !(l+r) !}=\frac{2^{2 r-1}}{r} \frac{(l !)^{2}}{(l-r) !(l+r) !}, \quad r \geq 1 .
$$

In order to evaluate the integral $J_{l+3 / 2}(\infty)$, again multiplying the identity (15) by $q_{l-r}^{2}$ with $\alpha=l+1 / 2$, integrating over a large interval $(0, c)$ and integration by parts, we have 


\section{WEAKLY ORTHOGONAL SPHERICAL HARMONICS}

$$
(2 l+1) J_{l+3 / 2}(c)-2 l J_{l+1 / 2}(c)=\left.\frac{q_{l-r}^{2} \lambda}{\left(1+\lambda^{2}\right)^{l+1 / 2}}\right|_{0} ^{c}-\int_{0}^{c} \frac{2 \lambda q_{l-r} q_{l-r}^{\prime}}{\left(1+\lambda^{2}\right)^{l+1 / 2}} d \lambda .
$$

As $c \rightarrow \infty$, the boundary limit vanishes for $r \geq 1$. By writing $\lambda q_{l-r}^{\prime}=(l-r) a_{l, l-r}^{(l-r)} \lambda^{l-r}+$ lots and using (14), we get $(2 l+1) J_{l+3 / 2}(\infty)-2 l J_{l+1 / 2}(\infty)=-2(l-r) J_{l+1 / 2}(\infty)$.

Hence,

$$
J_{l+3 / 2}(\infty)=\frac{2 r}{2 l+1} J_{l+1 / 2}(\infty)=\frac{2^{2 r}}{2 l+1} \frac{(l !)^{2}}{(l-r) !(l+r) !}, \quad r \geq 1 .
$$

Now, for the case $r=0$, the second terms in both sides of (16) become equal when $c \rightarrow \infty$ and get cancelled. However, the boundary limit becomes $a_{l, l}^{2}$. Thus we have $J_{l+3 / 2}(\infty)=\frac{\left(a_{l, l}^{(l)}\right)^{2}}{2 l+1}=\frac{1}{2 l+1}$. This is consistent with the case for $r \geq 1$.

Substituting these in (12), we have the inner product for the completely orthogonal spherical harmonics as

$$
\left\langle Z_{l, r}^{(k)}, Z_{l, r}^{(k)}\right\rangle=\frac{2^{2 r+1}}{2 l+1} \frac{(l !)^{2}}{(l+r) !(l-r) !}\left\{\begin{array}{ll}
2 \pi, & r=0 \\
\pi, & 0<r \leq l
\end{array}, l=0,1,2, \cdots, \quad k=0,1 .\right.
$$

\section{Linear relations and inner product}

In this section, we establish linear relations between the weakly orthogonal spherical harmonics $y_{l}^{(m, n)}$ and completely orthogonal spherical harmonics $Z_{l, r}^{(k)}$, and use these relations to obtain the inner product of the weakly orthogonal spherical harmonics.

By virtue of equations (3) and (8), it is enough to establish the relations between their corresponding polynomials $p_{l}^{(m, n)}$ and $Q_{l, r}^{(k)}$.

We define the column vector $\mathbb{P}_{1}$ of size $2 l+1$ of the polynomials $p_{l}^{(m, n)}, m+n=l-1, l$ for mode $l$ and the column vector of polynomials $\mathbb{Q}_{1}$ of the polynomials $Q_{l, r}^{(k)}, r=0,1, \cdots, l-k, k=0,1$. Let $S_{l}$ be the space of eigenfunctions $y_{l}^{(m, n)}, m+n=l-1, l$ for the face $X+$ on the sphere with the face inner product $\langle p, q\rangle_{X+}=\int_{-1}^{1} \int_{-1}^{1} p q s_{t}^{-3 / 2} d t_{1} d t_{2}$. Since the weakly orthogonal and completely orthogonal eigenfunctions are in the same eigenspace, they are linearly related. Since both set of eigenfunctions have the same factor $s_{t}^{-l / 2}$ with their corresponding polynomials, the polynomials are also linearly related with the same linear coefficients.

Moreover, since the spherical harmonics are either odd or even in the coordinate parameters $t_{1}, t_{2}$, the face inner product vanishes for two spherical harmonics components with different parities making them 'locally orthogonal'. Since there are four combinations of parity differences namely (ev,ev), (ev,od), (od,ev) and $(o d, o d)$ for the coordinate parameters, the spherical harmonics for a given mode $l$ can be grouped into four blocks of mutually locally orthogonal sets.

The above observation is valid for both weakly orthogonal and completely orthogonal spherical harmonics. The difference is that, for the completely orthogonal spherical harmonics, the functions within a set block are 


\section{M.A.A.M. FAHAM and H.M. NASIR}

also orthogonal (with respect to the inner product (7)), while the weakly orthogonal spherical harmonics are not, whence the name weakly orthogonal.

Define

$$
\begin{aligned}
& P_{l}^{(e v, k)}=\left\{p_{l}^{(m, n)}: m-\text { even }, m+n=l-k\right\}, P_{l}^{(o d, k)}=\left\{p_{l}^{(m, n)}: m-o d d, m+n=l-k\right\}, k=0,1, \\
& Q_{l}^{(e v, k)}=\left\{Z_{l, r}^{(k)}: m=\operatorname{deg}\left(t_{1}\right)-\text { even }, \operatorname{deg}\left(t_{2}\right)=l-m-k\right\} \\
& \text { and } Q_{l}^{(e v, k)}=\left\{Z_{l, r}^{(k)}: m=\operatorname{deg}\left(t_{1}\right)-o d d, \operatorname{deg}\left(t_{2}\right)=l-m-k\right\}
\end{aligned}
$$

so that the column vectors $\mathbb{P}_{l}$ and $\mathbb{Q}_{l}$ with their four blocks are $\mathbb{P}_{l}=\left[\mathbb{P}_{l}^{(e v, 0)} ; \mathbb{P}_{l}^{(o d, 0)} ; \mathbb{P}_{l}^{(e v, 1)} ; \mathbb{P}_{l}^{(o d, 1)}\right]$ and $\mathbb{Q}_{l}=\left[\mathbb{Q}_{l}^{(e v, 0)} ; \mathbb{Q}_{l}^{(o d, 0)} ; \mathbb{Q}_{l}^{(e v, 1)} ; \mathbb{Q}_{l}^{(o d, 1)}\right]$ respectively. Also let $\mathbb{Y}_{l}$ and $\mathbb{Z}_{l}$ be the column vectors of their spherical harmonics respectively. Any linear relations between the functions in $\mathbb{P}_{l}$ and $\mathbb{Q}_{l}$ are limited to the functions in their corresponding block counterparts as the blocks of different locations are mutually orthogonal, as observed above, and hence linearly independent.

The key points in establishing the linear relations are the leading coefficients of the polynomials involved. The polynomials $p_{l}^{(m, n)}$ have the leading coefficient $a_{l}^{(m, n)}$ corresponding to the higher degree term $t_{1}^{m} t_{2}^{n}$. Then, we may write $p_{l}^{(m, n)}=a_{l}^{(m, n)} t_{1}^{m} t_{2}^{n}+$ lots or

$$
t_{1}^{m} t_{2}^{n}=p_{l}^{(m, n)} / a_{l}^{(m, n)}+\text { lots }
$$

For the polynomials $Q_{l, r}^{(k)}$ corresponding to the orthogonal spherical harmonics, we have

$$
\begin{aligned}
Q_{l, r}^{(0)}+\mathrm{i} Q_{l, r}^{(1)} & =\left(p_{r}^{(0)}+\mathrm{i} p_{r}^{(1)}\right)^{r} \rho\left(t_{1}\right)^{l-r} q_{(l-r)}(l, \lambda)=\left(t_{1}+\mathrm{i}\right)^{r} \rho\left(t_{1}\right)^{l-r} \sum_{n=r_{1}}^{l-r} 2_{l, n} a_{l, r)}^{(l-r)} \lambda^{n} \\
& =\left(t_{1}+\mathrm{i}\right)^{r} \sum_{n=r_{1}}^{l-r} 2_{l, n} a_{l}^{(l-r)} t_{2}^{n}\left(1+t_{1}^{2}\right)^{(l-r-n) / 2} .
\end{aligned}
$$

Being $l-r-n$ even, expanding this and separating the real and imaginary parts, we see that $Q_{l, r}^{(k)}, k=0,1$ are polynomials of total degree $l-k$ (see also Table 1). We may write them, with their leading coefficients, as

$$
\begin{aligned}
& Q_{l, r}^{(0)}=\sum_{n=r_{1}}^{l-r} a_{l, n}^{(l-r)} t_{1}^{l-n} t_{2}^{n}+\text { lots } \\
& Q_{l, r}^{(1)}=r \sum_{n=r_{1}}^{l-r} 2_{l, n}^{(l-r)} t_{1}^{l-1-n} t_{2}^{n}+\text { lots } .
\end{aligned}
$$

Note that while $p_{l}^{(m, n)}\left(t_{1}, t_{2}\right)$ has only one leading coefficient, $Q_{l, r}^{(k)}\left(t_{1}, t_{2}\right)$ has many leading coefficients for highest total degree $l-k$. Substituting (18) and using the orthogonality of $p_{l}^{(m, n)}$ with lower order polynomials, we have

$$
\begin{aligned}
Q_{l, r}^{(0)} & =\sum_{n=r_{1}}^{l-r} 2\left(a_{l, n}^{(l-r)} / a_{l}^{(l-n, n)}\right) p_{l}^{(l-n, n)} \\
& =\sum_{n=r_{1}}^{l-r} 2 \frac{(-1)^{(l-r-n) / 2}}{2^{(l-r-n)}}\left(\begin{array}{c}
l-n \\
(l-r-n) / 2
\end{array}\right) p_{l}^{(l-n, n)}
\end{aligned}
$$




\section{WEAKLY ORTHOGONAL SPHERICAL HARMONICS}

and

$$
\begin{aligned}
Q_{l, r}^{(1)} & =r \sum_{n=r_{1}}^{l-r} 2\left(a_{l, n}^{(l-r)} / a_{l}^{(l-1-n, n)}\right) p_{l}^{(l-1-n, n)} \\
& =\sum_{n=r_{1}}^{l-r} 2 \frac{r}{l-n} \frac{(-1)^{(l-r-n) / 2}}{2^{(l-r-n)}}\left(\begin{array}{c}
l-n \\
(l-r-n) / 2
\end{array}\right) p_{l}^{(l-1-n, n)} .
\end{aligned}
$$

From this, we obtain the matrix form of the linear relations between the sets of polynomials and hence between the weakly and completely orthogonal spherical harmonics as

$$
\mathbb{Q}_{l}=\mathbb{T}_{l} \mathbb{P}_{l} \text { and } \mathbb{Z}_{l}=\mathbb{T}_{l} \mathbb{Y}_{l}
$$

respectively, where $\mathbb{T}_{l}$ is a square matrix of size $2 l+1$ consisting of the coefficients of the linear relations in (19) and (20). According to the choice of blocks of the column vectors $\mathbb{P}_{l}$ and $\mathbb{Q}_{l}$, the structure of the matrix $\mathbb{T}_{l}$ is given in the block diagonal matrix form

$$
\mathbb{T}_{l}=\operatorname{diag}\left[A_{l}, B_{l}, C_{l}, D_{l}\right],
$$

where $A_{l}, B_{l}, C_{l}$ and $D_{l}$ are upper triangular matrices with sizes $n_{A, l}=\lceil(l+1) / 2\rceil, \quad n_{B, l}=n_{C, l}=\lceil l / 2\rceil$ and $n_{D, l}=\lceil(l-1) / 2\rceil$ respectively, and with $\lceil x\rceil$ denoting the smallest integer such that $x \leq\lceil x\rceil$. The coefficients of the matrices are given by

$$
\begin{gathered}
A_{l}(i, j)=\left(-\frac{1}{4}\right)^{j-i}\left(\begin{array}{c}
2 j-2 \\
j-i
\end{array}\right), \quad B_{l}(i, j)=\left(-\frac{1}{4}\right)^{j-i}\left(\begin{array}{c}
2 j-1 \\
j-i
\end{array}\right), \\
C_{l}(i, j)=\frac{(2 i-1)}{(2 j-1)}\left(-\frac{1}{4}\right)^{j-i}\left(\begin{array}{c}
2 j-1 \\
j-i
\end{array}\right) \text { and } D_{l}(i, j)=\frac{i}{j}\left(-\frac{1}{4}\right)^{j-i}\left(\begin{array}{c}
2 j \\
j-i
\end{array}\right)
\end{gathered}
$$

respectively for $j \geq i$, and zeros otherwise. The indices $i, j$ range up to their respective matrix sizes. These coefficients can be obtained successively by the relations

$$
\begin{gathered}
A_{l}(1,1)=1, \quad A_{l}(i, j+1)=-\frac{1}{4} \frac{(2 j)(2 j-1)}{(j+1-i)(j+i-1)} A_{l}(i, j), \quad A_{l}(i+1, j)=-4 \frac{(j-i)}{i+j-1} A_{l}(i, j) \\
B_{l}(i, j)=\frac{2 j-1}{i+j-1} A_{l}(i, j), \quad C_{l}(i, j)=\frac{2 i-1}{i+j-1} A_{l}(i, j) \quad \text { and } \quad D_{l}(i, j)=\frac{i}{j} A_{l}(i+1, j+1)
\end{gathered}
$$

for their respective sizes.

It is also noted that for even mode $l \neq 0, A_{l}=A_{l+1}, B_{l-1}=B_{l}, C_{l-1}=C_{l}$ and $D_{l}=D_{l+1}$. When the matrices change sizes in alternative modes, their sizes increase by only one row and column. The entries of the row are all zero except the last, which is one. Therefore, by appending one column, we may obtain the matrices for the next mode. The coefficients of $B_{l}, C_{l}$ and $D_{l}$ can also be obtained from the simplified relations with constant coefficients as

$$
B_{l}(i, j)=A_{l}(i, j)-\frac{1}{4} A_{l}(i+1, j), C_{l}(i, j)=A_{l}(i, j)+\frac{1}{4} A_{l}(i+1, j), D_{l}(i, j)=2 B_{l}(i, j)-A_{l}(i+1, j+1) .
$$

The matrices, therefore, are obtained from the matrix $A_{l}$ only, and by using the above relations. The entries of matrix $A_{l}$ can be easily evaluated column wise by the recurrence formula

$$
A_{l}(j, j)=1, A(i-1, j)=-\frac{1}{4} \frac{j+i-2}{j-i+1} A_{l}(i, j), \quad i=j, j-1, \cdots, 1, \quad j=1,2, \cdots, n_{A, l} .
$$




\section{M.A.A.M. FAHAM and H.M. NASIR}

The inner product for the weakly orthogonal spherical harmonics is determined using the linear relations established between the two sets of spherical harmonics and the inner product of the completely orthogonal spherical harmonics.

Since the matrices in (22) are upper triangular matrices, they can be easily inverted. The relation between the inner products of the two sets of spherical harmonics is then given in matrix form as

$$
\left\langle\mathbb{Z}_{l}, \mathbb{Z}_{l}\right\rangle=\mathbb{Z}_{l} \mathbb{Z}_{l}^{T}=\mathbb{T}_{l} \mathbb{Y}_{l} \mathbb{Y}_{l}^{T} \mathbb{T}_{l}^{T}=\mathbb{T}_{l}\left\langle\mathbb{Y}_{l}, \mathbb{Y}_{l}\right\rangle \mathbb{T}_{l}^{T}
$$

from which we obtain the matrix of inner products for the weakly orthogonal spherical harmonics for model $l$ as

$$
\left\langle\mathbb{Y}_{l}, \mathbb{Y}_{l}\right\rangle=\mathbb{T}_{l}^{-1}\left\langle\mathbb{Z}_{l}, \mathbb{Z}_{l}\right\rangle \mathbb{T}_{l}^{-T}
$$

Note that the matrix of inner products for the completely orthogonal spherical harmonics is a diagonal matrix. Further, matrix $\mathbb{T}_{l}$ consists of upper triangular block matrices. They can be easily inverted.

\section{Spherical Fourier series on cubed-sphere}

The Fourier series of a spherical function $f\left(t_{1}, t_{2}\right)$ is written in terms of the weakly orthogonal spherical harmonics as

$$
f\left(t_{1}, t_{2}\right)=\sum_{i=0}^{\infty} M_{i} \mathbb{Y}_{i}
$$

where $M_{i}$ are row vectors of sizes $2 l+1$ of coefficients given by $\mathbb{M}_{i}=\left\langle f, \mathbb{Y}_{i}\right\rangle\left\langle\mathbb{Y}_{i}, \mathbb{Y}_{i}\right\rangle^{-1}$.

It is interesting to note that the inverse matrix of inner products $\left\langle\mathbb{Y}_{l}, \mathbb{Y}_{l}\right\rangle^{-1}$ can be evaluated without inverting the matrix $\mathbb{T}_{i}$ In fact, we have from (23) that

$$
\left\langle\mathbb{Y}_{l}, \mathbb{Y}_{l}\right\rangle^{-1}=\mathbb{T}_{l}^{T}\left\langle\mathbb{Z}_{l}, \mathbb{Z}_{l}\right\rangle^{-1} \mathbb{T}_{l}
$$

Since $\left\langle\mathbb{Z}_{l}, \mathbb{Z}_{l}\right\rangle$, being the matrix of inner products of completely orthogonal spherical harmonics for model $l$, is diagonal, its inverse is also diagonal with the reciprocals of the diagonal entries.

The vector of coefficients $\left\langle f, \mathbb{Y}_{l}\right\rangle$ consists of entries of the form $\langle f, y\rangle=\sum_{F \in\{X \pm, Y \pm, Z \pm\}}\left\langle f, y_{F}\right\rangle_{F}$, where $y=y_{l}^{(m, n)}$ and $y_{F}$ is its restriction on one face of the cubed-sphere. The local inner product is given by

$$
\left\langle f, y_{F}\right\rangle_{F}=\int_{-1}^{1} \int_{-1}^{1} f\left(t_{1}, t_{2}\right) y_{F}\left(t_{1}, t_{2}\right) s_{l}^{-3 / 2} d t_{1} d t_{2} \text {. }
$$

\section{Conclusion}

We have established linear relations between weakly orthogonal and orthogonal spherical harmonics and their inner products, and a power representation for the weakly orthogonal spherical harmonics. Spherical functions can be decomposed by Fourier series using the weakly orthogonal spherical harmonics. Application of these weakly orthogonal spherical harmonics in problems in areas of science, such as weather prediction models, is a subject of further research.

\section{References}

ARCHIBALD, M., EVANS, K.J., DRAKE, J. and WHITE III, J.B. 2011. Multi-wavelet Discontinuous Galerkin-Accelerated Exact linear Part (ELP) Method for the Shallow-Water Equations on the Cubed 


\section{WEAKLY ORTHOGONAL SPHERICAL HARMONICS}

sphere. Monthly Weather Review, 139(2): 457-473.

CHEN, C. and XIAO, F. 2008. Shallow Water Model on Cubed-Sphere by Multi-Moment Finite Volume Method. Journal of Computational Physics, 227(10): 5019-5044.

GÓMEZ, R., LEHNER, L., PAPADOPOULOS, P. and WINICOUR, J. 1997. The eth Formalism in Numerical Relativity. Classical and Quantum Gravity, 14: 977-990.

KRALL, T.H. and SHEFFER, I.M. 1967. Orthogonal Polynomials in two Variables. Annali di Matematica Pura ed Applicata, 76(4): 325-376.

KWON, K.H., LEE, J.K. and LITTLEJOHN, L.L. 2001. Orthogonal Polynomial Eigenfunctions of Second Order Partial Differential Equations. Transactions of the American Mathematical Society, 353(9): 36293647.

LAURITZEN, P.H., NAIR, R.D. and ULLRICH, P.A. 2010. A Conservative Semi-Lagrangian Multi-Tracer Transport Scheme (CSLAM) on the Cubed-Sphere grid. Journal of Computational Physics, 229(5): 14011424.

LEE, J.K. and LITTLEJOHN, L.L. 2006. A Construction of Real Weight Functions for Certain Orthogonal Polynomials in two Variables. Journal of Mathematical Analysis and Applications, 319(2): 475-493.

MACROBERT, T.M. 1967. Spherical Harmonics. Pergamon Press.

NASIR, H.M. 2007. Spherical Harmonics in a Non-Polar Co-ordinate System and Application to Fourier Series in 2-sphere. Mathematical Methods in the Applied Sciences, 30(14): 1843-1854.

NASIR, H.M. and KAKO, T. 2001. Fictitious Domain Methods for Structural-Acoustic Coupling Problem in Unbounded Region. Theoretical and Applied Mechanics, 50: 391-401.

NASIR, H.M., KAKO, T. and KOYAMA, D. 2003. A Mixed Type Finite Element Approximation for Radiation Problems Using Fictitious Domain Method. Journal of Computational and Applied Mathematics, 152: 377 392.

PHILLIPS, N.A. 1957. A Map Projection System Suitable for Large-Scale Numerical Weather Prediction. In Syono, S., ed., 75th Anniversary Volume, J. Meteorological Society of Japan, 35: 262-267.

REISSWIG, C., BISHOP, N.T., LAI, C.W., THORNBURG, J. and SZILÁGYI, B. 2007. Characteristic Evolutions in Numerical Relativity using Six Angular Patches. Classical and Quantum Gravity, 24: $237-$ 339.

ROCKMORE, D.N., KOSTELEC, P.J., HEALY, D.M. and MOORE, S. 2003. Ffts for 2-Sphere-Improvements and Variations. Journal of Fourier Analysis and Applications, 9(4): 341-385.

RONCHI, C., IACONO, R. and PAOLUCCI, P.S. 1996. The 'Cubed Sphere': A New Method for the Solution of Partial Differential Equations in Spherical Geometry. Journal of Computational Physics, 124: 93-114.

SWARTZRAUBER, P.N. 1979. On the Approximation of Discrete Scalar and Vector Functions on the Sphere. SIAM J. Numerical Analysis, 16(6): 934-949.

SWARTZRAUBER, P.N. 1981. The Approximation of Vector Functions and their Derivatives on the Sphere. SIAM J. Numerical Analysis, 18(2): 191-210.

Received 25 April 2011

Accepted 7 March 2012 